\title{
Dosage du LSD dans les phanères par chromatographie liquide couplée à la spectrométrie de masse ou par chromatographie gazeuse couplée à la spectrométrie de masse tandem
}

\section{Determination of LSD in human hair by liquid chromatography mass spectrometry or gas chromatography tandem mass spectrometry}

Marjorie CHEZE, François VAYSSETTE, Gilbert PÉPIN

Laboratoire d'Expertises TOXLAB, 7, rue Jacques Cartier - 75018 PARIS Tél : 0158592800 - Fax 0158592801 - e-mail : labtoxlab@aol.com

(Reçu le 10 avril 2001 ; accepté le 11 mai 2001)

\section{$R E ́ S U M E$}

Devant la recrudescence de la consommation de drogues de synthèse telles que l'ecstasy et le LSD, le toxicologue se retrouve parfois confronté à des problèmes de sensibilité. Tel est le cas du LSD, puissant hallucinogène agissant à des doses très faibles, qui est habituellement vendu sous forme de buvards ("Acides") imprégnés à $50 \mu \mathrm{g}$ en moyenne (de 10 à $100 \mu \mathrm{g})$. Les concentrations retrouvées dans les phanères sont de l'ordre du picogramme, rendant sa détection particulièrement difficile. Nous avons utilisé avec succès les colonnes LSD ImmunElute (Microgenics), récemment commercialisées en France, pour une extraction de haute spécificité associée à des techniques de détection suffisamment sensibles et spécifiques telles que la chromatographie liquide couplée à la spectrométrie de masse (CLHP-SM) et la chromatographie gazeuse couplée à la spectrométrie de masse tandem (CPG-SM-SM). Nous avons utilisés ces techniques dans un cas de polytoxicomanie mise en évidence dans les poils pubiens après consommation importante de LSD et pour lequel la concentration était de $26 \mathrm{pg} / \mathrm{mg}$. La sensibilité de la technique en CPG-SM-SM autorise la détection spécifique du LSD dans les phanères à des concentrations très basses $(0,5 \mathrm{pg} / \mathrm{mg})$.

MOTS-CLÉS

LSD, poils pubiens, Immunoaffinité.

\section{SUMMARY}

Growing consumption of illicit drugs such as ecstasy or LSD has confronted toxicologists with analytical sensitivity difficulties. LSD is a potent hallucinogenic compound which is active at low doses and usually sold as blotter papers (small stamps) soaked at an average of $50 \mu \mathrm{g}(10-100 \mu \mathrm{g})$. Consecutive concentrations in hair are in the picogram range that leads to detection difficulties. We successfully used LSD ImmunElute columns recently available in France, for a high specific extraction coupled to enough sensitive and specific analytical techniques such as liquid chromatography-mass spectrometry (LC-MS) and gas chromatographytandem mass spectrometry (GC-MS-MS). We report a case of drug addiction as demonstrated by hair analysis with a large consumption of LSD. LSD tested positive at $26 \mathrm{pg} / \mathrm{mg}$ in pubic hair. The high sensitivity of GC-MS-MS allows the specific detection of $L S D$ in hair at very low levels $(0.5 \mathrm{pg} / \mathrm{mg})$.

KEY-WORDS

LSD, pubic hair, Immunoaffinity. 


\section{Introduction}

Des modifications importantes du comportement des toxicomanes se sont manifestées ces dernières années. La chute de la consommation d'héroïne, due en partie aux programmes de substitution, a été accompagnée d'une hausse notable de la consommation de cocaïne et d'une généralisation de la consommation, outre du cannabis, des autres drogues de synthèse telles que l'ecstasy et le LSD (1) pour lesquels les saisies sont en nette hausse.

On assiste par ailleurs à une recrudescence des polytoxicomanies où les consommateurs associent plusieurs substances illicites ou les alternent (héroïne et cocaïne la semaine, LSD et ecstasy le week-end). Ces consommations souvent initialement "récréatives", peuvent conduire à une grave dépendance.

Le LSD, dérivé synthétique de l'acide lysergique extrait de l'ergot de seigle, est un puissant hallucinogène. Liquide incolore et inodore obtenu par synthèse avec de l'acide lysergique, de l'ammoniaque, de l'alcool éthylique et du gaz carbonique, il n'a plus aucune indication thérapeutique. Les quantités infimes nécessaires pour obtenir un effet hallucinogène expliquent qu'il soit vendu le plus souvent sous forme de buvards de moins d'un centimètre de côté (communément dénommés "Acides") imprégnés à des doses faibles, de l'ordre de 5 à $130 \mu \mathrm{g}$ avec une moyenne d'environ $50 \mu \mathrm{g}$. Le LSD désorganise la neurotransmission cérébrale. Après ingestion, la première phase conduit à des perturbations à dominance physique. Elle est suivie d'une phase psychodysleptique constituant le "voyage" proprement dit. La "descente" et l'asthénie consécutive durent de 1 à 2 jours (2). Les concentrations observées sont de l'ordre du nanogramme dans les fluides biologiques et du picogramme dans les phanères, rendant particulièrement difficile sa détection. De plus le LSD n'est pratiquement jamais pris de façon quotidienne régulière contrairement à d'autres drogues.

A ce jour, seuls 3 travaux de mise en évidence de LSD dans les phanères chez des personnes déclarant consommer ont été publiés. Les concentrations retrouvées par Nakahara et al. sont de 8 et $17 \mathrm{pg} / \mathrm{mg}$, mais présentaient des pics à hauteur du bruit de fond (3). Rörich et al. ont présenté un cas à $1 \mathrm{pg} / \mathrm{mg}$ en HPLC à détection de fluorescence (4) pour une exposition d'au moins trente buvards dans les deux mois précédent le prélèvement. A ce jour, les seules techniques proposées pour l'identification du LSD dans les cheveux ont été respectivement la chromatographie en phase gazeuse couplée à la spectrométrie de masse (CPG-SM) et la chromatographie liquide haute performance à détection de fluorescence (HPLC-Fluo) qui offre une bonne sensibilité de détection sans avoir la spécificité de la spec- trométrie de masse apportée par la CLHP-SM et la CPG-SM-SM.

Nous rapportons ici un cas de polytoxicomanie grave, mis en évidence par ces deux dernières techniques dans les phanères avec consommation avérée de LSD chez une personne interpellée en possession 1500 buvards.

\section{Matériel et méthode}

Un jeune homme de 29 ans est arrêté en région parisienne en possession de 1500 buvards de LSD, de 5 flacons de méthadone et de 20 grammes d'Opium. L'instruction nécessitait la mise en évidence d'une consommation régulière ou non de stupéfiants et notamment de LSD. Les poils pubiens ont été prélevés en maison d'arrêt par le Dr Pépin, Directeur du Laboratoire d'expertises Toxlab environ 20 jours après les faits.

Les poils pubiens sont décontaminés par trois bains successifs au dichlorométhane puis broyés extemporanément. A $100 \mathrm{mg}$ sont ajoutés $5 \mathrm{ng}$ de $\mathrm{LSD}-\mathrm{d}_{3}$ (Radian) comme étalon interne deutéré. L'extraction de la matrice est conduite par sonication méthanolique (4 heures à $50^{\circ} \mathrm{C}$ ). Les extraits sont filtrés avant d'être évaporés puis repris par $5 \mathrm{ml}$ de tampon phosphate $(0,1 \mathrm{M} \mathrm{pH6)}$ et $300 \mu \mathrm{l}$ d'albumine bovine (Ortho Clinical Diagnostic) avant d'être purifiés par immunoaffinité sur colonnes LSD ImmunElute (Microgenics, France). Cette technique d'extraction repose sur la reconnaissance spécifique du LSD par un anticorps monoclonal sous forme de résine liquide à ajouter à l'échantillon. Le lavage de la résine après fixation du LSD permet l'élimination de la plupart des interférences. Le LSD est extrait par dénaturation de la résine au méthanol. Cet extrait est évaporé puis repris directement par $15 \mu \mathrm{l}$ de méthanol pour injection de $5 \mu \mathrm{l}$ en CLHP-SM ou dérivé par $15 \mu \mathrm{l}$ de BSTFA (Regis) à $70^{\circ} \mathrm{C}$ pendant 20 minutes pour injection de $2 \mu \mathrm{l}$ en CPG-SM-SM.

L'analyse de routine est réalisée par chromatographie liquide couplée à la spectrométrie de masse. Le système chromatographique comporte un injecteur automatique AS 200 (Perkin Elmer) et deux micro pompes série 200 couplées à un spectromètre de masse API Sciex 165 muni d'un interface électrospray assisté pneumatiquement. La séparation chromatographique s'effectue sur colonne C18 Kromasil 150 x 1 mm i.d., $5 \mu \mathrm{m}$ d.p.(Higgins Analytical) munie d'une pré-colonne de $10 \mathrm{~mm}$ en mode isocratique à $75 \%$ de tampon formiate $5 \mathrm{mM}$ pH3 et $25 \%$.d'acétonitrile enrichi en $\mathrm{MeOH}$ pour un débit de $60 \mu \mathrm{l} / \mathrm{min}$. La détection se fait en mode positif avec une tension d'orifice de $+40 \mathrm{~V}$ et une tension de spray de $+5,8 \mathrm{kV}$ en mode $\mathrm{SIM}$ à $\mathrm{m} / \mathrm{z}$ 
327.3 (LSD-d3), 324.3 (LSD) pour la quantification et $\mathrm{m} / 2223.2$ (LSD) pour la confirmation.

Pour une meilleure sensibilité et spécificité la chromatographie gazeuse couplée à la spectrométrie de masse tandem apparaît comme une excellente alternative. Le système est constitué d'une CPG HP 5890 Série II plus munie d'une colonne CP-Sil-8-CB $30 \mathrm{~m}$ x 0,25 mm i.d., $0,25 \mu \mathrm{m}$ e.f. (Chrompack). La programmation de température est la suivante : $80^{\circ} \mathrm{C}$ pendant 2 minutes suivi d'un gradient de $15^{\circ} \mathrm{C} /$ min jusqu'à $310^{\circ} \mathrm{C}$, température finale maintenue 6 minutes. Le spectromètre de masse est un Finnigan Mat TSQ 7000 de chez Thermoquest. L'ionisation se fait en mode impact électronique et la collision de filiation se fait à une pression d'argon de 1,8 mTorr pour une énergie de $-17 \mathrm{eV}$. L'acquisition se fait en mode SRM (Selected Reaction
Monitoring) à $\mathrm{m} / \mathrm{z} 253$ et 297 ions fils du $\mathrm{m} / \mathrm{z} 398$ (LSD-d3) et $\mathrm{m} / \mathrm{z}$ 253et 294 ions fils du m/z 395 (LSD). La détermination des autres stupéfiants a été réalisée selon des méthodes précédemment publiées (5-9).

\section{Résultats}

Cette méthode a été appliquée avec succès à un cas médico-légal et a permis de mettre en évidence la présence de LSD dans les poils pubiens d'un jeune consommateur avéré à la concentration de $26 \mathrm{pg} / \mathrm{mg}$ par CLHP-SM (figure 1 et 2), que nous avons confirmé à $29 \mathrm{pg} / \mathrm{mg}$ par CPG-SM-SM (figure 3). La consommation de LSD déclarée était de 15 buvards par semaine, soit une forte consommation, l'usage étant de un à trois buvards par soirée $(3 \times 5=15)$.

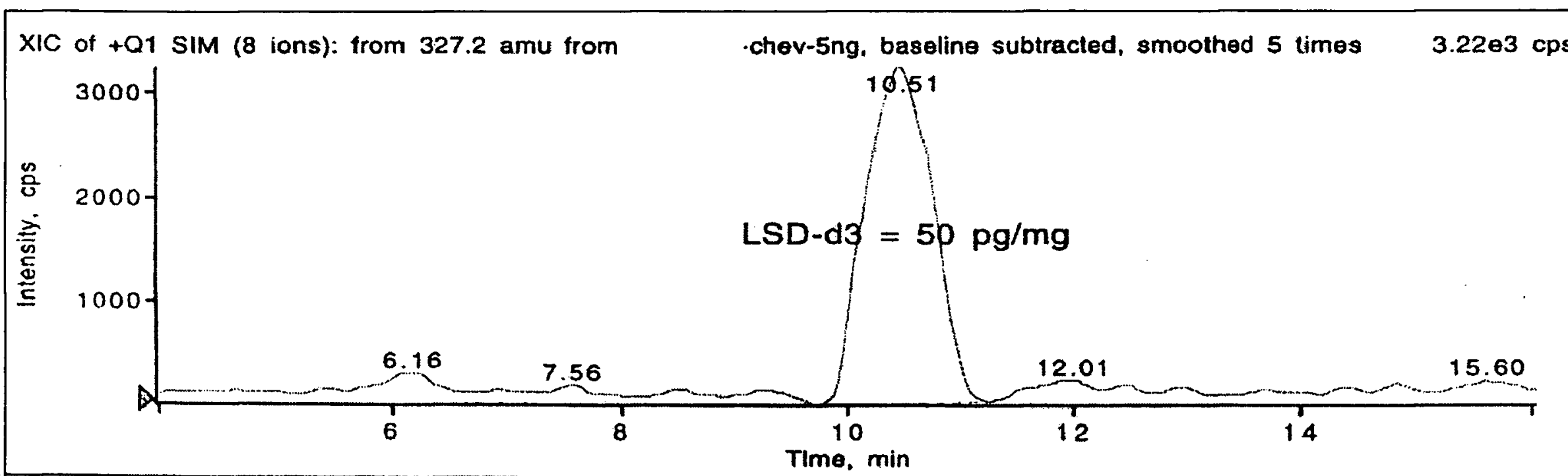

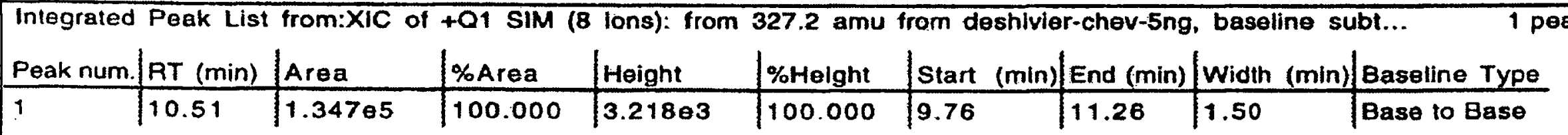

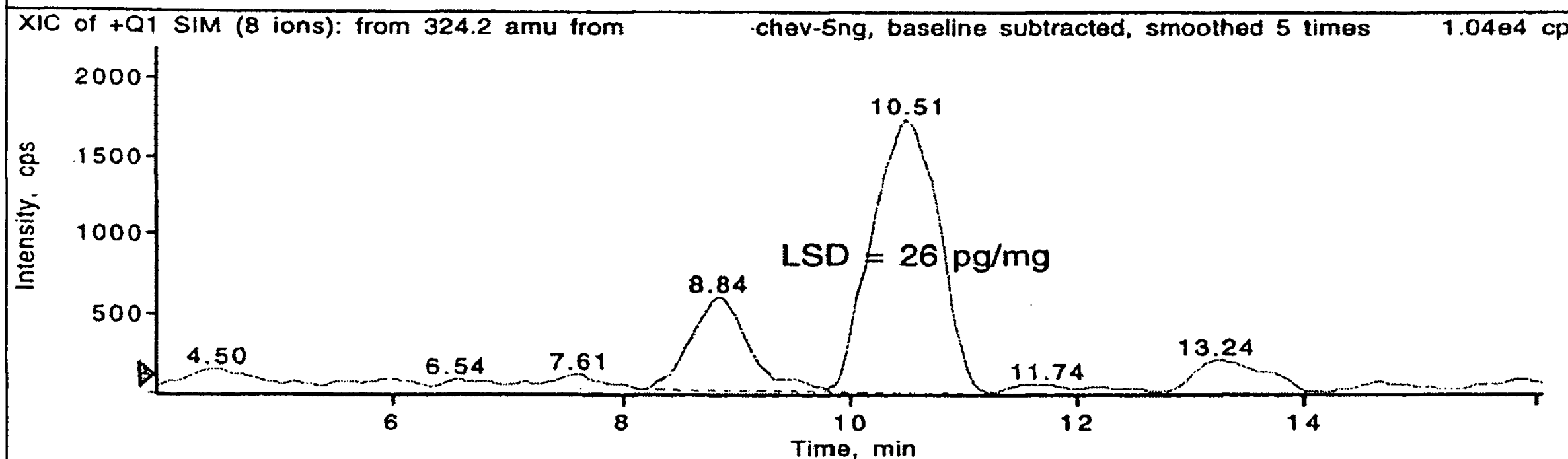

\begin{tabular}{|c|c|c|c|c|c|c|c|c|c|}
\hline $\begin{array}{l}\text { Integrated } \\
\text { Peak num. }\end{array}$ & $\begin{array}{l}\text { Peak List } \\
\text { RT (min) }\end{array}$ & from:XIC & $\begin{array}{l}\text { +Q1 SIM } \\
\% \text { Area }\end{array}$ & Hoight & $\begin{array}{l}324.2 \text { amt } \\
\% \text { Height }\end{array}$ & $\begin{array}{l}\text { rom deshivie } \\
\text { Start (min) }\end{array}$ & $\begin{array}{l}\text { r-chev-5ng } \\
\text { End (min) }\end{array}$ & $\begin{array}{l}\text { Waseline se } \\
\text { Width (min) }\end{array}$ & Ubt... \\
\hline 1 & 10.51 & 7.05204 & 100.000 & 1.71703 & 100.000 & 9.81 & 11.26 & 1.45 & Valley \\
\hline
\end{tabular}

Figure 1 : Chromatogramme CLHP-SM en mode SIM du LSD-d3 à $50 \mathrm{pg} / \mathrm{mg}$ (étalon interne à $\mathrm{m} / \mathrm{z} 327.3$ ) et du LSD à $26 \mathrm{pg} / \mathrm{mg}$ $(\mathrm{m} / \mathrm{z}$ 324.3) d'un extrait de poils pubiens d'un polytoxicomane. 


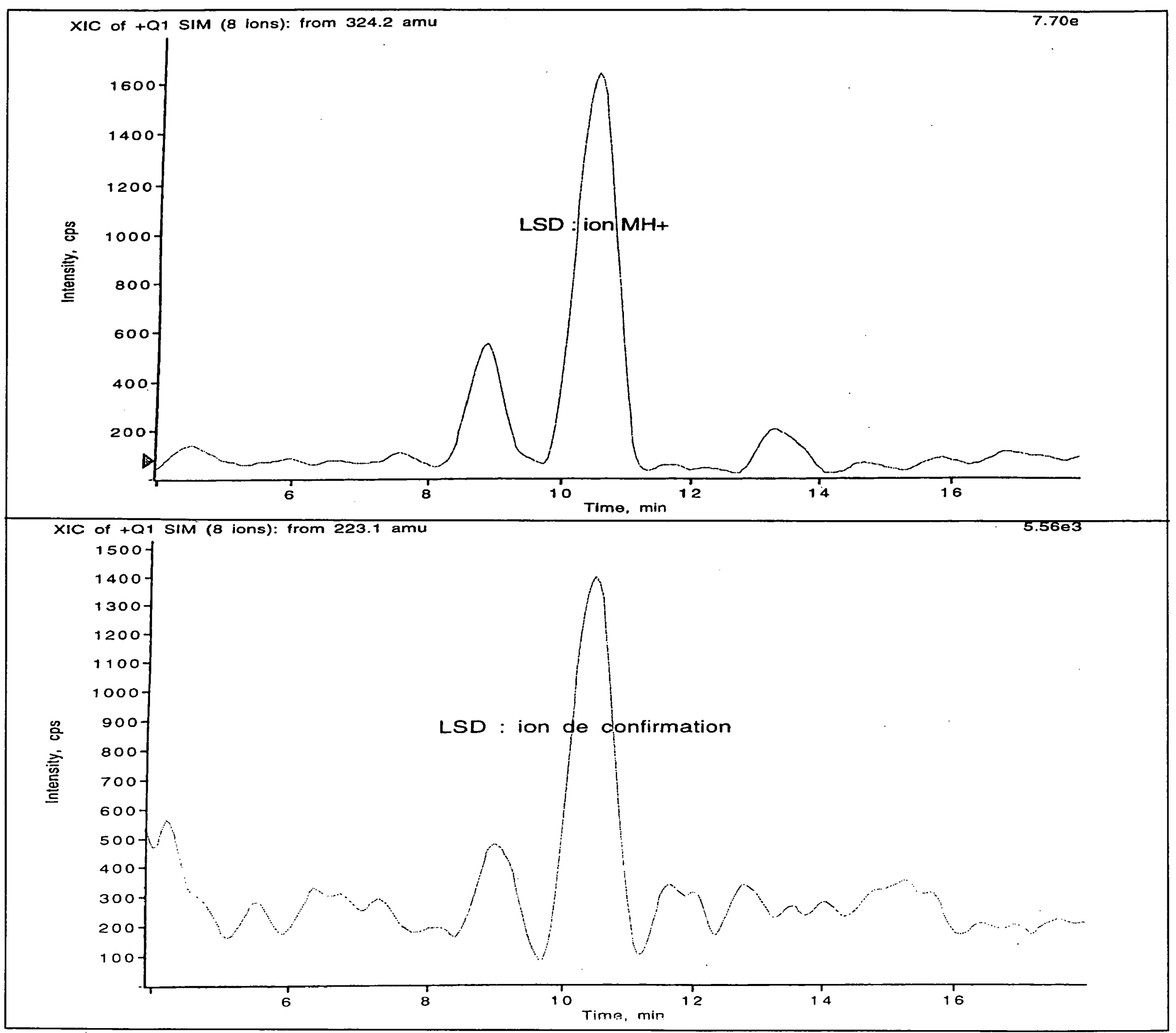

Figure 2 : Chromatogramme CLHP-SM en mode SIM de l'ion pseudomoléculaire $(\mathrm{m} / z$ 324.3) et de l'ion fragment de confirmation ( $\mathrm{m} / \mathrm{z} 223.3$ ) du LSD à $26 \mathrm{pg} / \mathrm{mg}$ d'un extrait de poils pubiens d'un polytoxicomane.

La technique d'extraction par immunoaffinité (LSD ImmunElute) offre des rendements de l'ordre de $80 \%$ avec une spécificité inégalée conduisant à une grande pureté des extraits. Les limites de détection sont de $4 \mathrm{pg} / \mathrm{mg}$ en CLHP-SM et de $0,5 \mathrm{pg} / \mathrm{mg}$ en CPG-SMSM. La reproductibilité n'a pas été étudiée compte tenu du coût des colonnes d'extraction. Cependant la corrélation des résultats obtenus sur le cas présenté par CLHP-SM et CPG-SM-SM est très satisfaisante. De plus, la nombreuse documentation concernant le dosage du LSD dans les fluides biologiques indique une bonne linéarité de réponse du LSD en CLHP-SM $(11,12,13)$.

Comme le montre le tableau I, la présence de nom- breux autres stupéfiants et toxiques (héroïne, méthadone, cocaïne, alcool, MDMA, cannabis) a été mise en évidence dans les poils pubiens de ce jeune homme de 28 ans. Ce cas, un peu atypique, illustre bien l'usage de nombreuses substances comme étant là tendance actuelle des toxicomanes ainsi que leur association à l'alcool mise en évidence par la présence de cocaéthylène (caractérisant la consommation simultanée de cocaïne et d'alcool).

La dépendance physique engendrée par le LSD est presque nulle. A long terme cependant, les gros consommateurs peuvent être atteints de désordres psychiques graves et irréversibles nécessitant une prise en charge psychiatrique comme cela a été le cas de ce jeune homme rapidement interné. 


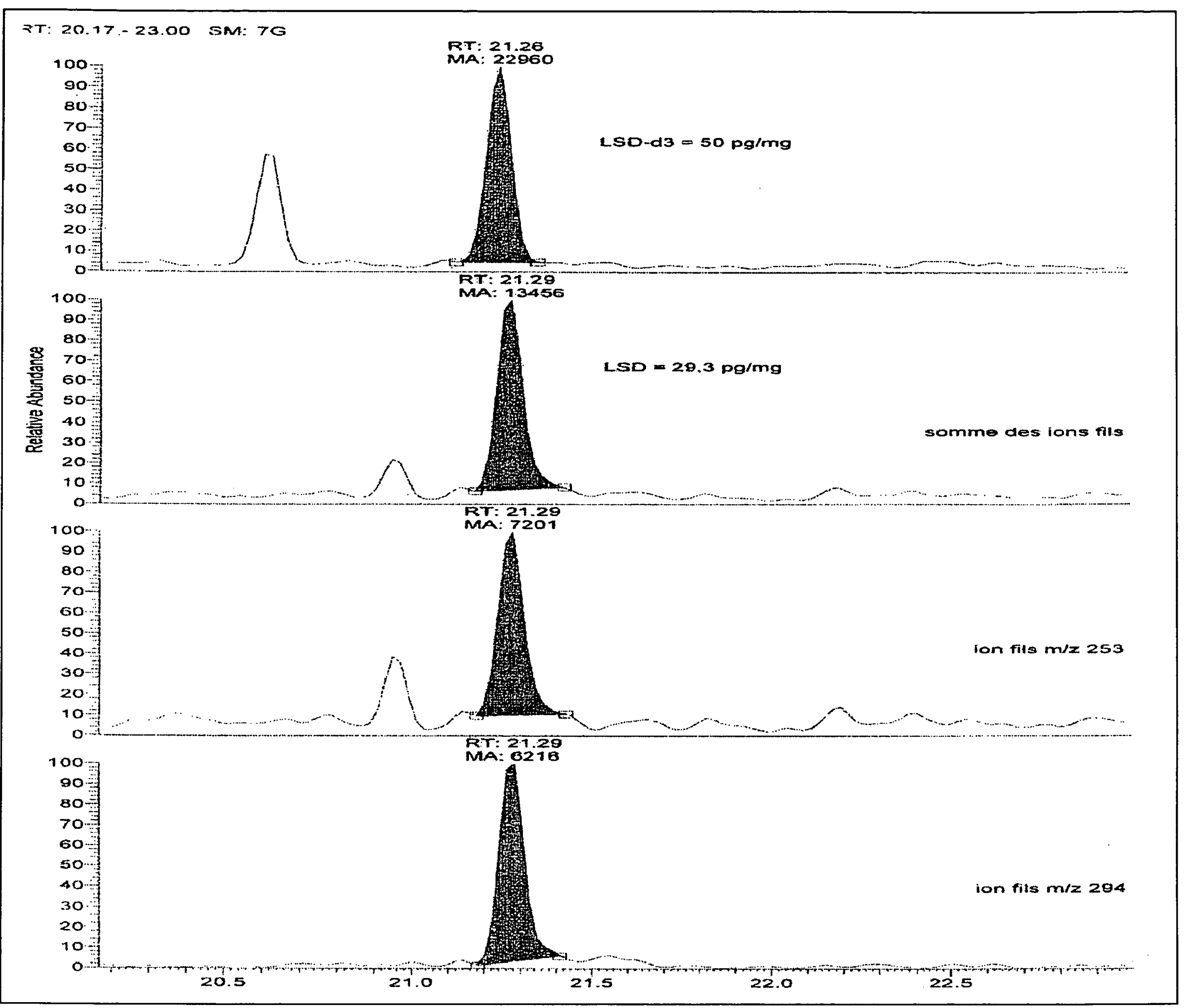

Figure 3 : Chromatogramme CPG-SM-SM en mode SRM des ions fils du LSD-d3 à $50 \mathrm{pg} / \mathrm{mg}$ (étalon interne à $\mathrm{m} / z$ 398.0) et du LSD à $29 \mathrm{pg} / \mathrm{mg}(\mathrm{m} / \mathrm{z} 395.0)$ d'un extrait de poils pubiens d'un polytoxicomane.

\section{Discussion}

Contrairement à la détection des opiacés, des cocaïniques, des amphétamines et des cannabinoïdes, la détection du LSD dans les phanères est très peu documentée. Son mécanisme d'incorporation au cheveu a été étudié par Nakahara et al. (10). Celui-ci a montré son affinité importante pour la mélanine laissant prévoir une bonne fixation, compensant les faibles quantités absorbées. Par ailleurs le LSD étant dégradé à la lumière UV directe et à un $\mathrm{pH}$ acide (11) sa détection semble plus indiquée dans les poils pubiens que dans les cheveux dans le cas d'une consommation régulière. A contrario, la détection des métabolites du LSD dans les phanères n'a pas été étudiée probablement dû au faible nombre de cas disponibles. On peut estimer cependant que son principal métabolite, le 2-oxo-3hydroxy-LSD, étant polaire sera faiblement fixé. De plus, son extraction sur colonnes d'immunoaffinité LSD Immun Elute n'est pas décrite.

Contrairement au cannabis où la détection des métabolites est particulièrement intéressante du fait de la contamination passive par la fumée, ce phénomène semble à priori être exclu pour le LSD dans les cheveux de part son mode même de consommation. Cependant la contamination "passive" des poils pubiens par l'urine par manque d'hygiène a été observée pour d'autres drogues et ne peut être exclue pour le LSD. Elle n'implique bien sûr que le consommateur lui-même mais peut tromper sur la corrélation entre les concentrations observées et l'importance de la consommation. 
Tableau I : Concentrations en stupéfiants dans les poils pubiens d'un polytoxicomane.

\begin{tabular}{|c|c|}
\hline Produits retrouvés & $\begin{array}{c}\text { Concentration dans } \\
\text { les poils pubiens } \\
\text { en } \mathbf{~ g / m g}\end{array}$ \\
\hline cocaine & 78,1 \\
\hline benzoylecgonine & 38,8 \\
\hline methyl ecgonine & $<0,5$ \\
\hline cocaéthylène & 1,9 \\
\hline monoacétylmorphine & 9,2 \\
\hline morphine & 8,4 \\
\hline codéine & 3,1 \\
\hline méthadone & 12,9 \\
\hline tétrahydrocannabinol & 1,1 \\
\hline cannabinol & positif \\
\hline cannabidiol & positif \\
\hline acide tétrahydrocannabinoïque & 0,007 \\
\hline MDMA & 0,65 \\
\hline LSD & 0,027 \\
\hline
\end{tabular}

\section{Conclusion}

Une extraction de haute spécificité utilisant les colonnes LSD ImmunElute (Microgenics, France) dédiées au départ à l'urine associée à la chromatographie liquide couplée à la spectrométrie de masse (CLHP-SM) apporte une réponse satisfaisante pour la recherche de LSD en première intention dans les phanères chez des consommateurs réguliers. De part sa simplicité, sa spécificité, la rapidité de sa mise en œuvre et sa limite de détection cette technique apporte une solution satisfaisante. Cependant la chromatographie gazeuse couplée à la spectrométrie de masse tandem (CPG-SM-SM), bien qu'onéreuse, est une alternative intéressante de part sa haute spécificité nécessaire en analyse toxicologique médico-légale, et sa grande sensibilité qui laisse prévoir la mise en évidence dans les phanères de consommations bien moindres, environ 40 à 50 fois inférieure à celle présentée ici.

Ces techniques devraient permettre d'enrichir l'arsenal analytique du toxicologue face à la nouvelle émergence de consommation de LSD dont la fenêtre de détection limitée à quelques heures dans l'urine est amenée à plusieurs semaines voire plusieurs mois par l'analyse des phanères.

\section{Références}

1. Tracqui A., Kintz P., Doray S., Geraut A., Ludes B., Actualité du LSD en 1997. J. Méd. Lég. 1998 ; 41, 3-4: 240-243.

2. Vincent F. : Hallucinogènes. In : Kintz P., ed. Toxicologie et pharmacologie médico-légales. Paris : Elsevier, 1998 : 560-561.

3. Nakahara Y., Kikura R., Takahashi K., Foltz R.L., Mieczkowski T., Detection of LSD and metabolite in rat hair and human hair. J. Anal. Toxicol. 1996 ; 20, 5 : 323329.

4. Rohrich J., Zorntlein S., Becker J., Analysis of LSD in human body fluids and hair samples applying ImmunElute columns. For. Sci. Int. $2000 ; 107$ : 181-190.

5. Kintz P., Cirimele V., Mangin P., Testing human hair for cannabis. II. Identification of THC-COOH by GC/MSNCI as an unique proof. J. Forensic Sci. 1995 ; 40 : 619622.

6. Cirimele V., Sachs H., Kintz P., Mangin P., Testing human hair for cannabis. III. Rapid screening procedure for simultaneous identification of D9-tetrahydrocannabinol, cannabinol and cannabidiol. J. Anal. Toxicol. $1996 ; 20$ : 13-16.

7. Gaillard Y., Pepin G., Testing hair for pharmaceuticals. J. Chromatogr. B. $1999 ; 733: 231-246$.
8. Gaillard Y., Pepin G., Simultaneous solid-phase extraction on $\mathrm{C}_{18}$ cartridges of opiates and cocainics for an improved quantitation in human hair by GC-MS : one year of forensic applications. Forensic Sci. Int. 1997 ; $86: 49-59$.

9. Moeller M.R., Fey P., Wenning R., Simultaneous determination of drugs of abuse (opiates, cocaïne, and amphetamine) in human hair by GC/MS and its application to a methadone treatment program. Forensic Sci. Int. 1993 ; $63: 185$.

10.Nakahara Y., Takahashi K., Kikura R., Hair analysis for drugs of abuse. X. Effect of physicochemical properties of drugs on the incorporation rates into hair. Biol. Pharma. Bull. 1995 ; 18, 9 : 1223-1227.

11. Verstraete A. Dosage sérique et urinaire du LSD. Toxicorama. 1998 ; X, 3 : 122-125.

12.Sauvage M.F., Marquet P., Ragot S., Lachatre F., Dupuy J.L., Lachatre G., Dosage du LSD et de trois de ses métabolites ou isomères dans le sérum et le sang total par LCES-MS. Toxicorama. $1998 ; \mathrm{X}, 2$ : 73-79.

13.Hoja A., Marquet P., Verneuil B., Lotfi H., Dupuy J.L., Lachatre G., Determination of LSD and N-demethyl-LSD in urine by liquid chromatography coupled to electrospray ionization mass spectrometry. J. Chromatogr. B. 1997 ; 692 : 329-335. 